\title{
AN INVOLUTION INEQUALITY FOR THE KULLBACK-LEIBLER DIVERGENCE
}

\author{
IOSIF PINELIS
}

Abstract. Let $R_{t}:=t P+(1-t) P t$, where $t \in(0,1), P$ is a probability measure, and $P t$ is the push-forward image of $P$ under a measurable involution $\imath$. An inequality involving the Kullback-Leibler divergence of $R_{t}$ from $P$ is given. It is shown that the role of the involution is essential.

Mathematics subject classification (2010): Primary 26D10, 26D15; Secondary 16W10, 26A48, 46Kxx, 60E15, 62B10, 94A17.

distributions.

Keywords and phrases: Kullback-Leibler divergence, involution, reflection, inequalities, mixtures of

\section{REFERENCES}

[1] An inequality of KL divergence, 2015, MathOverflow, http://mathoverflow.net/questions/ 222482/an-inequality-of-kl-divergence/222560\#222560.

[2] S. Kullback AND R. A. Leibler On information and sufficiency, Ann. Math. Statistics, 22: 79-86, 1951. 